\title{
巨型多不确定串匹配完全自动机及其快速生成 算法
}

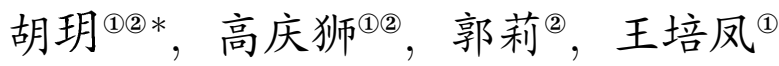

(1) 北京科技大学信息工程学院, 北京 100083

(2) 中国科学院计算技术研究所, 北京 100190

* 通信作者. E-mail: huhuyue_001@sina.com

摘要 在串匹配搜索中, 字符串常常采用 U- 不确定串、V-不确定串及其结合的 U-V-不确定串. 如 何识别巨量 U- 不确定字符串、V-不确定字符串和 U-V- 不确定字符串, 以及两个和两个以上 U-V不确定字符串的交错情况的串匹配, 是没有遗漏地检测有害信息的关键问题. 本文提出一个快速检 测巨量 U- 不确定字符串、巨量 V- 不确定字符串和巨量 U-V-不确定字符串的多串匹配完全自动机 及其快速生成方法, 包括两个和两个以上不确定字符串相互交错的情况; 并且给出 V- 不确定字符串 的完全自动机的最大并行台数, 指出通常正则表达式匹配可能出现相似连接和交错情况的两种遗漏, 指出如果没有从整体的角度对 U- 不确定串中的字符子串集进行两两不相交化及无同源后续奇点化 的处理, 结果就可能出现错误或者增加状态数目.

关键词多串匹配 U- 不确定串 V-不确定串 U-V-不确定串 完全自动机

\section{1 前言}

串匹配是计算机科学的经典问题, 它广泛应用于模式识别、拼写检查、自然语言处理, 指令检测、 垃圾防范、搜索引擎、防止病毒入侵、蠕虫围堵和生物信息处理．快速串匹配算法近半个世纪已经 被许多计算机研究者广泛研究 ${ }^{[1-25]}$. Aho 和 Corasick ${ }^{[1]}$ 提出了多串匹配自动机, 但不是完全自动机. Navarro 和 Raffinot 在文献 [2] 中总结了 2001 年及其之前的有关串匹配的研究成果, 并且在介绍 Aho 和 Corasick 工作的同时, 提出了多串匹配完全自动机 (所有的状态都根据各种不同输入转换成为其新 的状态) 的思想, 但没有进一步讨论其具体内容和有关的算法. 文献 [26] 提出一个生成 N-R 完全自动 机的具体 5 步 $O(u m k)$ 方法, 其中 $u$ 符号集中符号的个数, $k$ 和 $m$ 是串的个数和平均长度 (参见第 2 节).

不确定串匹配有两种类型. 一种是 U- 不确定串, 其中每个字符不是确定字符, 而是一个字符子集 合中的任意一个字符; 另一种是 $\mathrm{V}$ - 不确定串, 每个串中的字符之间可能夹杂与该串无关的 $k$ 个字符, 其中 $k$ 小于等于某个整数 $K$. 
虽然正则表达式可以处理前者和部分后者, 但是处理前者时效率比较低; 处理后者, 不能全部有 效. 例如, 从 $A_{1} A_{2} B_{1} B_{2} A_{3} B_{3} A_{4} B_{4} A_{5}$ 检测出 $A_{1} A_{2} A_{3} A_{4}$ 和 $B_{1} B_{2} B_{3} B_{4}$ 来, 就是一个不能全部有效处 理的例子. 已有处理正则表达式方法都不能处理相似子连接 ${ }^{[26]}$, 而且在交错情况时可能出现遗漏.

本文第 2 节简要叙述快速生成多串匹配完全自动机的方法, 高效快速生成巨量多 U- 不确定串、 V不确定串完全自动机的方法和 U-V- 不确定串多串匹配完全自动机算法分别在第 3-5 节讨论, 最后是 结束语.

\section{2 快速生成多串匹配完全自动机五步法的简述}

定义 1 对应输入字母表中的任意一个字母, 所有的状态都有一个相应确定的转移, 而且不需要 经过任何计算的自动机被称作为完全自动机.

快速生成多串匹配完全自动机可以通过以下 5 步生成 ${ }^{[26]}$ :

1) 符号串集的符号串排序;

2) 形成 $B_{i^{-}}$结构树;

3) $B_{i^{-}}$结构树节点的编码;

4) 带有根节点的子串与另一个不带根节点的子串相似状态转换的补充连接;

5) 补充连接与状态连接补全.

例如, 符号串集 $\{\mathrm{ABCD}, \mathrm{EFABC}, \mathrm{ADC}, \mathrm{ABDE}, \mathrm{ERAB}, \mathrm{BCE}, \mathrm{DE}\}$. 通过排序, 形成 $B_{i^{-}}$结构树及 第 3 步编码之后, 得到

$$
\begin{aligned}
& \mathrm{A}(1)(\mathrm{B}(2)(\mathrm{C}(3) \mathrm{D}(4), \mathrm{D}(5) \mathrm{E}(6)), \mathrm{D}(7) \mathrm{C}(8)) ; \mathrm{B}(9) \mathrm{C}(10) \mathrm{E}(11) ; \mathrm{D}(12) \mathrm{E}(13) ; \\
& \mathrm{E}(14)(\mathrm{F}(15) \mathrm{A}(16) \mathrm{B}(17) \mathrm{C}(18), \mathrm{R}(19) \mathrm{A}(20) \mathrm{B}(21)),
\end{aligned}
$$

如表 1 所示.

第 3 步之后, 连接是不完全的, 必须加上第 4 步相似连接, 如表 2 所示.

第 5 步是所有可能的连接都补全, 生成完全自动机.

\section{3 快速生成 U- 不确定串多串匹配完全自动机算法}

设, 给定一个字符集 $\Omega$ 和一个字符串子集的集 $\Sigma=\left\{A_{1}, A_{2}, \ldots, A_{n}\right\}$, 其中, 字符串子集 $A_{i}=\left\{R_{i 1}\right.$, $\left.R_{i 2}, \ldots, R_{i n_{i}}\right\}, R_{i j}=C_{i j_{1}} C_{i j_{2}} \ldots C_{i j n_{i j}}, C_{i j_{k}} \in \Omega, 1 \leqslant k \leqslant n_{i j}, 1 \leqslant j \leqslant n_{i}, 1 \leqslant i \leqslant n$.

定义 $2 \mathrm{U}$ - 不确定串 $U$ 是一个由字符串子集 $U_{i}$ 构成的串, $U=U_{1} U_{2} \ldots U_{m}$, 其中, $U_{i} \in \Sigma$, 其 中, 每个字符串子集 $U_{i}$ 不是确定的字符串, 而是一个字符串子集 $\left\{R_{i 1}, R_{i 2}, \ldots, R_{i n_{i}}\right\}$ 中的任意一个字 符串 $R_{i j}, R_{i j}=C_{i j_{1}} C_{i j_{2}} \cdots C_{i j n_{i j}}, C_{i j_{k}} \in \Omega$.

例如, 由 $\{$ 封, F, 丰, 峰 $\},\{$ 伸, shen, sh, 深, 神 $\},\{$ 榜, Ban, 棒 $\}$ 构成的 U- 不确定串: 封 shen 榜, $\mathrm{F}$ 神榜, 风深榜, 等等. 其中 $U_{1}=\{$ 封, $\mathrm{F}$, 丰, 峰 $\}, U_{2}=\{$ 伸, shen, sh, 深, 神 $\}, U_{3}=\{$ 榜, Ban, 棒 $\}$.

定义 3 (1) 如果 $U$ - 不确定串 $U$ 中存在在一个字符串子集 $U_{i}=\left\{R_{i 1}, R_{i 2}, \ldots, R_{i n_{i}}\right\}$ 中有两个字 符串 $\left(R_{i j}, R_{i j^{\prime}}\right)$ 存在 “包含” 关系, 则称该 $\mathrm{U}$ - 不确定串 $U$ 中的 $U_{i}$ 存在 “奇点”.

(2) 如果 $\mathrm{U}$ - 不确定串 $U=U_{1} U_{2} \cdots U_{m}$ 中的 $U_{i}=\left\{A_{1}, A_{2}, \ldots, A_{n}\right\}, U_{i+1}=\left\{B_{1}, B_{2}, \ldots, B_{n}\right\}$, 存在 一对字符串子集 $A_{i} \in\left\{R_{i 1}, R_{i 2}, \ldots, R_{i n_{i}}\right\}$ 及 $B_{t} \in\left\{R_{t 1}, R_{t 2}, \ldots, R_{t n_{t}}\right\}$, 满足 $R_{i j}=R_{i j^{\prime}}+R_{t k}$, 则称该 
表 1 第 3 步编码之后

Table 1 After coding in Step 3

\begin{tabular}{|c|c|c|}
\hline Original state & Input symbol & New state \\
\hline 0 & $\mathrm{~A}, \mathrm{~B}, \mathrm{D}, \mathrm{E}$ & $1,9,12,14$ \\
\hline 1 & $\mathrm{~B}, \mathrm{D}$ & 2,7 \\
\hline 2 & C, D & 3,5 \\
\hline 3 & $\mathrm{D}$ & $\underline{4}$ \\
\hline \multicolumn{3}{|l|}{4} \\
\hline 5 & $\mathrm{E}$ & $\underline{6}$ \\
\hline \multicolumn{3}{|l|}{6} \\
\hline 7 & $\mathrm{C}$ & $\underline{8}$ \\
\hline \multicolumn{3}{|l|}{8} \\
\hline 9 & $\mathrm{C}$ & 10 \\
\hline 10 & $\mathrm{E}$ & $\underline{11}$ \\
\hline \multicolumn{3}{|l|}{11} \\
\hline 12 & $\mathrm{E}$ & $\underline{13}$ \\
\hline \multicolumn{3}{|l|}{13} \\
\hline 14 & $\mathrm{~F}, \mathrm{R}$ & 15,19 \\
\hline 15 & $\mathrm{~A}$ & 16 \\
\hline 16 & B & 17 \\
\hline 17 & $\mathrm{C}$ & $\underline{18}$ \\
\hline \multicolumn{3}{|l|}{18} \\
\hline 19 & $\mathrm{~A}$ & 20 \\
\hline 20 & B & $\underline{21}$ \\
\hline 21 & & \\
\hline
\end{tabular}

表 2 第 4 步相似连接之后

Table 2 After supplement connection in Step 4

\begin{tabular}{|c|c|c|}
\hline Original state & Input symbol & New state \\
\hline 0 & $\mathrm{~A}, \mathrm{~B}, \mathrm{D}, \mathrm{E}$ & $1,9,12,14$ \\
\hline 1 & $\mathrm{~B}, \mathrm{D}$ & 2,7 \\
\hline 2 & C, D & 3,5 \\
\hline 3 & $\mathrm{D}, \mathrm{E}$ & $\underline{4}, \underline{11}$ \\
\hline 4 & $\mathrm{E}$ & $\underline{13}$ \\
\hline 5 & $\mathrm{E}$ & $\underline{6}-\underline{13}$ \\
\hline 6 & $\mathrm{~F}$ & 15 \\
\hline 7 & $\mathrm{C}$ & $\underline{8}$ \\
\hline \multicolumn{3}{|l|}{8} \\
\hline 9 & $\mathrm{C}$ & 10 \\
\hline 10 & $\mathrm{E}$ & $\underline{11}$ \\
\hline 11 & $\mathrm{~F}, \mathrm{R}$ & 15,19 \\
\hline 12 & $\mathrm{E}$ & $\underline{13}$ \\
\hline 13 & $\mathrm{~F}$ & 15 \\
\hline 14 & $\mathrm{~F}, \mathrm{R}$ & 15,19 \\
\hline 15 & $\mathrm{~A}$ & 16 \\
\hline 16 & B & 17 \\
\hline 17 & $\mathrm{C}$ & $\underline{18}$ \\
\hline 18 & $\mathrm{D}$ & $\underline{4}$ \\
\hline 19 & $\mathrm{~A}$ & 20 \\
\hline 21 & B & $\underline{21}$ \\
\hline 21 & $\mathrm{C}$ & 3 \\
\hline
\end{tabular}

$\mathrm{U}$ - 不确定串 $U=U_{1} U_{2} \cdots U_{m}$ 中的 $U_{i}$ 存在 “同源后续奇点”. 例如, U- 不确定串 $U=U_{1} U_{2} U_{3}$, $U_{1}=\left\{A_{1}, \mathrm{CD}, \mathrm{CDE}\right\}, U_{2}=\{\mathrm{E}\}, U_{3}=\{\mathrm{F}, \mathrm{G}\}$, 存在同源后续奇点.

以下讨论的多串匹配完全自动机的输入符串都假设是 U- 不确定串.

\subsection{U- 不确定串中的字符子串集两两不相交而且没有同源后续奇点}

定理 1 U- 不确定串中的字符子串集两两不相交而且没有同源后续奇点情况下, 可以生成多串 匹配完全自动机.

证明 假设, $\mathrm{U}$ - 不确定串 $U=U_{1} U_{2} \cdots U_{m}$ 的字符串子集 $A_{i}=\left\{R_{i 1}, R_{i 2}, \ldots, R_{i n_{i}}\right\}, 1 \leqslant i \leqslant n$, 两两不相交, 而且没有同源后续奇点.

取 U- 不确定串的字符子串集 $A_{i}$ 分别对应于字符 $F_{i}$.

考虑由输入字符 $F_{i}, i=1,2, \ldots, n$, 构成的多串匹配的完全自动机. 该自动机可以由第 2 节所讨论 的 5 步算法生成.

然后用 $\left\{\left(R_{i j}, T_{i}\right) \mid j=1,2, \ldots, n_{i}\right\}$ 个状态变换代替状态变换 $\left(F_{i}, T_{i}\right)$. 证毕.

显然, 当 $R_{i j}=C_{i j_{1}} C_{i j_{2}} \cdots C_{i j n_{i j}}, C_{i j_{k}}, n_{i j}>1$ 时状态 $T_{i}$ 的数量需要增加, 即添加中间过程变换的 状态. 
例如, $\{$ 榜, $\mathrm{B}$, 棒 $\}: T_{1}$ 状态下, 输入为 “榜”, 或者 “B”, 或者 “棒” 都转到 $T_{2}$ 状态.

而, $\{$ 榜, Ban, 棒 $\}: T_{1}$ 状态下, 输入为 “榜”, 或者 “棒” 都转到 $T_{2}$ 状态, 输入为 “B” 转入 $T_{3} ; T_{3}$ 状态下, 输入为 “a” 转入 $T_{4} ; T_{4}$ 状态下, 输入为 “n” 才转入 $T_{2}$; 增加了两个状态.

\subsection{U- 不确定串中的字符子串集非两两不相交或者有同源后续奇点}

定理 2 U- 不确定串中的字符子串集非两两不相交或者有同源后续奇点情况下, 可以先化为两 两不相交且无同源后续奇点, 然后生成多串匹配完全自动机.

证明 假设, U- 不确定串的字符子串集 $A_{i}=\left\{R_{i 1}, R_{i 2}, \ldots, R_{i n_{i}}\right\}, 1 \leqslant i \leqslant n$, 非两两不相交, 或者 有同源后续奇点; 令, 把交集独立成为一个字符子串集 $A_{i}^{\prime}$, 原一个字符子串集去掉交集部分成为新的 字符子串集, 这个过程一直进行下去, 直到全部字符子串集成为两两不相交为止.

再把有奇点的字符子串集 $A_{i}^{\prime}$ 的奇点单独成为一个字符子串集 $A_{I}^{\prime \prime}$, 原字符子串集 $A_{i}^{\prime}$ 去掉奇点成 为一个新字符子串集 $A_{I}^{\prime \prime}$, 这个过程一直进行下去, 直到全部字符子串集没有奇点为止.

把 U- 不确定串 $U=U_{1} U_{2} \cdots U_{m}$ 中存在同源后续奇点的 $U_{i}=\left\{A_{1}, A_{2}, \ldots, A_{n}\right\}$ 和 $U_{i+1}=$ $\left\{B_{1}, B_{2}, \ldots, B_{n}\right\}$, 满足 $R_{p j}=R_{p j^{\prime}}+R_{t k}$ 的 $U_{i}$ 中把 $R_{p j}$ 分离出来成为一个独立的新字符子串集 $A_{p^{\prime}}$, 其中, $A_{i} \in\left\{R_{i 1}, R_{i 2}, \ldots, R_{i n_{i}}\right\}, B_{t} \in\left\{R_{t 1}, R_{t 2}, \ldots, R_{t n_{t}}\right\}$, 这个过程一直进行下去, 直到全部字符 子串集没有同源后续奇点为止.

最后, 由第 2 节所讨论的 5 步算法生成多串匹配的完全自动机. 证毕.

例 $1\{\{$ 榜, B, Ban, 棒 $\},\{$ 榜, Ba, Ban, 旁 $\}\}$, 化为两两不相交且无同源后续奇点的: $\{\{$ 榜, Ban $\},\{\mathrm{B}$, 棒 $\},\{\mathrm{Ba}$, 旁 $\}\}$.

例 $2\{\{$ 榜, Ba, Ban, 棒 $\},\{$ 榜, Ba, Ban, 旁 $\}\}$, 化为两两不相交的: $\{\{$ 榜, Ba, Ban $\},\{$ 棒 $\},\{$ 旁 $\}\}$; 然 后再化为两两不相交且无同源后续奇点的: $\{\{$ 榜, Ban $\},\{\mathrm{Ba}\},\{$ 棒 $\},\{$ 旁 $\}\}$.

\section{4 快速生成 V- 不确定串多串匹配完全自动机算法}

设, 给定一个字符集 $\Omega$ 和一个字符串子集的串集 $\Pi=\left\{S_{1}, S_{2}, \ldots, S_{m}\right\}$, 其中, $S_{i}=C_{i 1} C_{i 2} \cdots C_{i n_{i}}$, $C_{i j} \in \Omega, 1 \leqslant j \leqslant n_{i}, 1 \leqslant i \leqslant n$.

定义 $4 \mathrm{~V}$ - 不确定串是由 $V_{i}$ 构成的串, $\mathrm{V}=V_{1} V_{2} \cdots V_{n}$, 其中, $V_{i} \in \Omega, i=1,2, \ldots, n$; 如果存在一 个 $S_{i} \in \Pi, S_{i}=C_{i 1} C_{i 2} \cdots C_{i m}$, 满足 $m \leqslant n, C_{i 1}=V_{j 1}, C_{i 2}=V_{j 2}, \ldots, C_{i m}=V_{j m}$; 其中, $1 \leqslant j_{1} \leqslant j_{2} \leqslant$ $\cdots \leqslant j_{m} \leqslant n$; 即称 $\mathrm{V}=V_{1} V_{2} \ldots V_{n}$, 为 $S_{i}$ 的 $\mathrm{V}$ - 不确定串.

例如, “ $C_{1} C_{2} b_{1} b_{2} C_{3} b_{3} C_{4} b_{4} C_{5}$ ” 是字符串 $S_{i}=C_{1} C_{2} C_{3} C_{4} C_{5}$ 的 V- 不确定串, 其中, $C_{i} \in \Omega, b_{j} \in \Omega$.

\subsection{V- 不确定串多串匹配完全自动机的自动生成算法}

算法 $1 \mathrm{~V}$ - 不确定串多串匹配完全自动机的自动生成算法.

1) 先考虑在字符集 $\Omega$ 上的字符串子集的串集 $\Pi$, 由第 2 节所讨论的 5 步算法中的方法生成完全 自动机 (包括第 4 步 “相似连接”).

例如, 符号串集: $\{\mathrm{ABCD}, \mathrm{EFABC}, \mathrm{ADC}, \mathrm{ABDE}, \mathrm{ERAB}, \mathrm{BCE}, \mathrm{DE}\}$. 通过排序, 形成 $B_{i^{-}}$结构树 及第 3 步编码之后, 得到

$$
\begin{aligned}
& \mathrm{A}(1)(\mathrm{B}(2)(\mathrm{C}(3) \mathrm{D}(4), \mathrm{D}(5) \mathrm{E}(6)), \mathrm{D}(7) \mathrm{C}(8)) ; \mathrm{B}(9) \mathrm{C}(10) \mathrm{E}(11) ; \mathrm{D}(12) \mathrm{E}(13) ; \\
& \mathrm{E}(14)(\mathrm{F}(15) \mathrm{A}(16) \mathrm{B}(17) \mathrm{C}(18), \mathrm{R}(19) \mathrm{A}(20) \mathrm{B}(21)),
\end{aligned}
$$


参见表 1.

加上第 4 步相似连接, 如表 2 所示.

2) 设置一个计数器, 计数器有两个输入 (“置确定值” 和 “减 1 ”) 和 1 个状态输出 (“0”).

3) 由于任何两个状态 (假设为 $n_{1}, n_{x}$ ) 之间的输入符 (假设为 $\mathrm{X}$ ) 之前可能出现其他字符 (假设为 $\mathrm{Y}$, 这是 V- 不确定串所允许的), 而且这些字符不影响输入符 (X) 之后的状态 $\left(n_{x}\right)$;

(i) 令状态 $n_{1}$ 之后的任意除了 $\mathrm{X}$ 之外的输入符之后的状态为非 $n_{x}$ 的 $n_{2}$, 并且计数器 “减 1 ”;

(ii) 令状态 $n_{2}$ 之后除了 $\mathrm{X}$ 之外的任意输入符之后的状态仍然为 $n_{2}$, 并且计数器 “减 1 ”;

(iii) 如果状态 $n_{1}$ 之后的输入为 $\mathrm{X}$, 状态转到 $n_{x}$, 则令计数器 “置确定值”; 如果该输入 $\mathrm{X}$ 在 0 - 状 态输入时是转向 “初始状态”, 则同时令一台副完全自动机并行地启动, 进入初始状态;

(iv) 如果状态 $n_{1}$ 之后的输入为非 “X”, 而且计数器为 0 , 则令转向状态为 0 或者 “初始状态”.

以上, “初始状态” 指 “从 0 状态根据输入字符转向” 的非 0 状态.

\section{$4.2 \mathrm{~V}$ - 不确定字符串多串匹配需要多台并行工作的完全自动机}

定理 3 使用多台并行工作的完全自动机, 可以处理多个交错出现的 V- 不确定字符串.

证明 以下采用构造性证明.

1) 多个 V- 不确定字符串可能交错出现.

一个字符串的字符之间可能出现另一个字符串的字符, 即, 两个或者两个以上的字符串交错; 完全 自动机必须检测它们.

2) 处理多个交错出现的 V- 不确定字符串需要多台并行工作的完全自动机, 其中首先使用的完全 自动机是主完全自动机, 其他的是 “副完全自动机”.

3) 上面 (iii) 提到, 当非 0 状态 $n_{1}$ 之后的输入 (X), 状态转到非 0 状态 $n_{X}$ 时; 如果该输入 (X) 在 0 - 状态输入时是转向 “初始状态”, 则同时并行地启动一台副完全自动机进入初始状态.

4) 一个字符串的字符之间也可能出现非 “另一个字符串的字符” 的字符; 各完全自动机也同样必 须检测它们.

5) 并行工作的完全自动机的台数为可能交错出现的字符串数目. 即, 小于等于计数器的确定值, 也就是, 一个字符串的字符之间可能出现的其他字符长度.

6 ) 副完全自动机转移到 0 - 状态等于关闭该副完全自动机. 主完全自动机转移到 0 - 状态就是转移 到 0 - 状态本身.

\section{5 快速生成 U-V- 不确定串多串匹配完全自动机算法}

设, 给定一个字符集 $\Omega$, 一个字符串子集的集 $\Sigma=\left\{A_{1}, A_{2}, \ldots, A_{n}\right\}$ 和一个字符串子集的串集 $\Pi$ $=\left\{S_{1}, S_{2}, \ldots, S_{m}\right\}$, 其中, 字符串子集的串 $S_{t}=A_{t 1} A_{t 2} \cdots A_{t n_{i t}}, A_{t j} \in \Sigma$; 不妨假设, $A_{i}, i=1,2, \ldots, n$, 两两不相交而且没有同源后续奇点 (否则需要先经过 3.2 小节的两两不相交化和无同源后续奇点化的 处理).

字符串子集 $A_{i}=\left\{R_{i 1}, R_{i 2}, \ldots, R_{i n_{i}}\right\}, R_{i j}=C_{i j_{1}} C_{i j_{2}} \cdots C_{i j n_{i j}}, C_{i j_{k}} \in \Omega, 1 \leqslant k \leqslant n_{i j}, 1 \leqslant j \leqslant n_{i}$, $1 \leqslant i \leqslant n$.

考虑 $W=W_{1}, W_{2}, \ldots, W_{L}$, 如果存在一个 $S_{t} \in \Pi, S_{t}=A_{t 1} A_{t 2} \cdots A_{t m}$, 满足 $m \leqslant L, A_{i 1}=$ $W_{j 1}, A_{i 2}=W_{j 2}, \ldots, A_{i m}=W_{j m}$; 其中, $1 \leqslant j_{1} \leqslant j_{2} \leqslant \cdots \leqslant j_{m} \leqslant L$; 其他的 $W_{i}=B_{i}, B_{i}=$ 
$\left\{D_{i 1}, D_{i 2}, \ldots, D_{i n_{i}}\right\}, D_{i j}=\left\{E_{i j_{1}} E_{i j_{2}} \cdots E_{i j n_{i j}}\right\}, E_{i j_{k}} \in \Omega, j=1,2, \ldots, n_{i}, k=1,2, \ldots, n_{i j} . \quad$ 即称 $W$ 为 $S_{i}$ 的 $\mathrm{U}-\mathrm{V}-$ 不确定串.

例如, “ $A_{1} B_{1} A_{2} B_{2} A_{3}$ ” 是字符串 $S_{i}=A_{1} A_{2} A_{3}$ 的 U-V- 不确定串, 其中, $A_{i} \in \Sigma, B_{j}=\left\{b_{j 1}, b_{j 2}, \ldots\right.$, $\left.b_{j n_{j}}\right\}, b_{j k} \in \Omega, i=1,2,3,1 \leqslant j \leqslant 2,1 \leqslant k \leqslant n_{j} . A_{1}=\{$ 封, F, 丰, 峰 $\}, A_{2}=\{$ 伸, shen, sh, 深, 神 $\}$, $A_{3}=\{$ 榜, Ban, 棒 $\}$. 即, “\{封, F, 丰, 峰 $\} B_{1}\{$ 伸, shen, sh, 深, 神 $\} B_{2}\{$ 榜, Ban, 棒 $\}$ ”构成的 U-V-不确

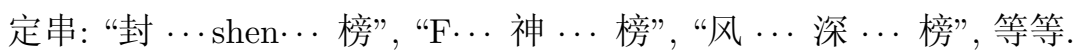

\subsection{U-V- 不确定串多串匹配完全自动机的快速生成算法}

算法 2 快速生成 U-V- 不确定串多串匹配完全自动机算法.

1) 首先生成 U- 不确定串多串匹配完全自动机:

因为字符串集 $\left(A_{i}\right)$ 两两不相交而且没有同源后续奇点, 所以可以用字符 $\left(F_{i}\right)$ 来代替字符串集 $\left(A_{i}\right)$, 因此, 可以采用第 2 节所讨论的 5 步法来生成完全自动机 (见 3.1 小节).

2) 然后生成 V- 不确定串多串匹配完全自动机 (同前):

(i) 设置一个计数器, 计数器有两个输入 (“置确定值” 和 “减 1 ”) 和 1 个状态输出 (“0”).

(ii) 由于任何两个状态 (假设为 $n_{1}, n_{X}$ ) 之间的输入符 (假设为 $\mathrm{X}$ ) 之前可能出现其他字符 (假设 为 $\mathrm{Y}$, 这是 $\mathrm{V}$ - 不确定串所允许的), 而且这些字符不影响输入符 $(\mathrm{X})$ 之后的状态 $\left(n_{X}\right)$.

令, 状态 $n_{1}$ 之后的任意除了 $\mathrm{X}$ 之外的输入符之后的状态为 $n_{2}$, 并且计数器 “减 1 ”, 状态 $n_{2}$ 之后 除了 $\mathrm{X}$ 之外的任意输入符之后的状态仍然为 $n_{2}$, 并且计数器 “减 1 ”; 如果状态 $n_{1}$ 之后的输入为 $\mathrm{X}$, 则 “置确定值” 计数器, 并且状态转到 $n_{X}$; 如果状态 $n_{1}$ 之后的输入为非 $\mathrm{X}$, 而且计数器为 0 , 则转向 0 状态或者 “初始状态”. 其中, “初始状态”指 “从 0 状态根据输入字符转向” 的非 0 状态.

(iii) 需要并行工作的多完全自动机 (即, 需要除了主完全自动机之外的第 2 个、第 3 个、…副 自动机).

如果状态 $n_{1}$ 之后的输入为非 $\mathrm{X}$, 而且计数器为 0 , 则转向 0 状态或者 “初始状态” 的同时, 如果 输入符在 0 状态时, 转向一个初始状态, 则还需要同时并行地启动该初始状态. (即, 启动主完全自动 机之外的一台副自动机.)

(iv) 副完全自动机上转移到 0 状态等于关闭该自动机.

\section{2 问题讨论 1: 多 U-V- 不确定串的交错}

多 U-V- 不确定串也可能交错.

1) 多 V- 不确定串的交错. 例如, V- 不确定串 “ $A_{1} B_{1} A_{2} C_{1} B_{2} C_{2} A_{3} C_{3} B_{3}$ ” 是 3 个 V-不确定串的 交错, 其中, 字符串 $S_{1}=A_{1} A_{2} A_{3}, S_{2}=B_{1} B_{2} B_{3}, S_{3}=C_{1} C_{2} C_{3}, A_{i} \in \Omega, B_{i} \in \Omega, C_{i} \in \Omega, i=1,2,3, \Omega$ 是 给定的符号集.

2) 多 U-V- 不确定串的交错. 例如, U-V- 不确定串 “ $A_{1} B_{1} A_{2} C_{1} B_{2} C_{2} A_{3} C_{3} B_{3}$ ” 是 3 个 U-V- 不确 定串的交错, 其中, U- 不确定字符串 $S_{1}=A_{1} A_{2} A_{3}, S_{2}=B_{1} B_{2} B_{3}, S_{3}=C_{1} C_{2} C_{3}, A_{i} \in \Sigma, B_{i} \in \Sigma, C_{i} \in \Sigma$, $i=1,2,3, \Sigma$ 是两两不相交而且没有同源后续奇点的 (由符号集 $\Omega$ 中的字符组成的) 字符串集的集.

\section{3 问题讨论 2: U- V- 不确定串多串匹配需要并行工作的多完全自动机台数}

显然, $V-$ 不确定串和 $U-V-$ 不确定串的多串匹配需要并行工作的副完全自动机的台数是小于等于 被匹配的字符串中两个字符之间允许间隔的长度. 


\section{4 问题讨论 3: 正则表达式匹配可能的遗漏}

虽然孤立的 U-V- 不确定串可以使用正则表达式来表示. 但是, 直接使用通常正则表达式匹配可 能出现相似连接的遗漏和交错情况的遗漏; 而且没有从整体的角度对 U- 不确定串中的字符子串集进 行两两不相交化及无同源后续奇点化的处理, 结果, 就可能出现错误或者相当大地增加状态数目.

\subsection{U-V- 不确定串多串匹配完全自动机的快速生成算法的一个例子}

一个例子: “ $A_{1} B_{1} A_{2} B_{2} A_{3}$ ” 是字符串 $S_{1}=A_{1} A_{2} A_{3}$ 的 U-V- 不确定串, “ $A_{4} B_{3} A_{5} B_{4} A_{6} B_{5} A_{7}$ ” 是字符 串 $S_{2}=A_{4} A_{5} A_{6} A_{7}$ 的 U-V- 不确定串, 其中, $A_{i} \in \Sigma, B_{i}=\left\{b_{i 1}, b_{i 2}, \ldots, b_{i n_{i}}\right\}, b_{i j} \in \Omega, i=1,2,3, \ldots, 1 \leqslant$ $j \leqslant n_{j} ; A_{1}=\{$ 封, F, Fen, 丰, 峰 $\}, A_{2}=\{$ 伸, shen, sh, 深, 神 $\}, A_{3}=\{$ 榜, B, Ba, Ban, 棒 $\} ; A_{4}=\{$ 榜, B, Ba, Ban, 邦, 斑 $\}, A_{5}=\{$ 德, de, 的, d $\}, A_{6}=\{$ 故, Gu, G, 固 $\}, A_{7}=\{$ 事, $\mathrm{S}$, 实, Sh $\}$; 即, “ “封, F, 丰, 峰 $\} B_{1}\{$ 伸, shen, sh, 深, 神 $\} B_{2}\{$ 榜, Ban, 棒 $\}$ ”构成的 U-V- 不确定串, “封 …shen …榜”, “F … 神 ... 榜”, “风 ... 深.... 榜”, 等等.

1) 进行无关化及无同源后续奇点化: $A_{1}^{\prime}=\{$ 封, F, 丰, 峰 $\}, A_{2}^{\prime}=\{\mathrm{Fen}\}, A_{3}^{\prime}=\{$ 伸, shen, 深, 神 $\}$, $A_{4}^{\prime}=\{\mathrm{sh}\}, A_{5}^{\prime}=\{$ 榜, B $\}, A_{6}^{\prime}=\{\mathrm{Ba}\}, A_{7}^{\prime}=\{\mathrm{Ban}\}, A_{8}^{\prime}=\{$ 棒 $\}, A_{9}^{\prime}=\{$ 邦, 斑 $\}, A_{10}^{\prime}=\{$ 德, 的, d $\}, A_{11}^{\prime}=\{\mathrm{de}\}$, $A_{12}^{\prime}=\{$ 故, G, 固 $\}, A_{13}^{\prime}=\{\mathrm{Gu}\}, A_{14}^{\prime}=\{$ 事, $\mathrm{S}$, 实 $\}, A_{15}^{\prime}=\{\mathrm{Sh}\}$; 得

$$
\begin{aligned}
S_{1}= & A_{1} A_{2} A_{3}=\left(A_{1}^{\prime}, A_{2}^{\prime}\right)\left(A_{3}^{\prime}, A_{4}^{\prime}\right)\left(A_{5}^{\prime}, A_{6}^{\prime}, A_{7}^{\prime}, A_{8}^{\prime}\right) \\
= & A_{1}^{\prime}\left(A_{3}^{\prime}\left(A_{5}^{\prime}, A_{6}^{\prime}, A_{7}^{\prime}, A_{8}^{\prime}\right), A_{4}^{\prime}\left(A_{5}^{\prime}, A_{6}^{\prime}, A_{7}^{\prime}, A_{8}^{\prime}\right)\right), A_{2}^{\prime}\left(A_{3}^{\prime}\left(A_{5}^{\prime}, A_{6}^{\prime}, A_{7}^{\prime}, A_{8}^{\prime}\right), A_{4}^{\prime}\left(A_{5}^{\prime}, A_{6}^{\prime}, A_{7}^{\prime}, A_{8}^{\prime}\right)\right), \\
S_{2}= & A_{4} A_{5} A_{6} A_{7}=\left(A_{5}^{\prime}, A_{6}^{\prime}, A_{7}^{\prime}, A_{9}^{\prime}\right)\left(A_{10}^{\prime}, A_{11}^{\prime}\right)\left(A_{12}^{\prime}, A_{13}^{\prime}\right)\left(A_{14}^{\prime}, A_{15}^{\prime}\right) \\
= & A_{5}^{\prime}\left(A_{10}^{\prime}\left(A_{12}^{\prime}\left(A_{14}^{\prime}, A_{15}^{\prime}\right), A_{13}^{\prime}\left(A_{14}^{\prime}, A_{15}^{\prime}\right)\right), A_{11}^{\prime}\left(A_{12}^{\prime}\left(A_{14}^{\prime}, A_{15}^{\prime}\right), A_{13}^{\prime}\left(A_{14}^{\prime}, A_{15}^{\prime}\right)\right)\right), \\
& A_{6}^{\prime}\left(A_{10}^{\prime}\left(A_{12}^{\prime}\left(A_{14}^{\prime}, A_{15}^{\prime}\right), A_{13}^{\prime}\left(A_{14}^{\prime}, A_{15}^{\prime}\right)\right), A_{11}^{\prime}\left(A_{12}^{\prime}\left(A_{14}^{\prime}, A_{15}^{\prime}\right), A_{13}^{\prime}\left(A_{14}^{\prime}, A_{15}^{\prime}\right)\right)\right), \\
& A_{7}^{\prime}\left(A_{10}^{\prime}\left(A_{12}^{\prime}\left(A_{14}^{\prime}, A_{15}^{\prime}\right), A_{13}^{\prime}\left(A_{14}^{\prime}, A_{15}^{\prime}\right)\right), A_{11}^{\prime}\left(A_{12}^{\prime}\left(A_{14}^{\prime}, A_{15}^{\prime}\right), A_{13}^{\prime}\left(A_{14}^{\prime}, A_{15}^{\prime}\right)\right)\right), \\
& A_{9}^{\prime}\left(A_{10}^{\prime}\left(A_{12}^{\prime}\left(A_{14}^{\prime}, A_{15}^{\prime}\right), A_{13}^{\prime}\left(A_{14}^{\prime}, A_{15}^{\prime}\right)\right), A_{11}^{\prime}\left(A_{12}^{\prime}\left(A_{14}^{\prime}, A_{15}^{\prime}\right), A_{13}^{\prime}\left(A_{14}^{\prime}, A_{15}^{\prime}\right)\right)\right) .
\end{aligned}
$$

2) 第 3 步后得

$$
\begin{gathered}
S_{1}=(0) A_{1}^{\prime}(1)\left(A_{3}^{\prime}(2)\left(A_{5}^{\prime}(3), A_{6}^{\prime}(4), A_{7}^{\prime}(5), A_{8}^{\prime}(6)\right), A_{4}^{\prime}(7)\left(A_{5}^{\prime}(8), A_{6}^{\prime}(9), A_{7}^{\prime}(10), A_{8}^{\prime}(11)\right)\right), \\
A_{2}^{\prime}(12)\left(A_{3}^{\prime}(13)\left(A_{5}^{\prime}(14), A_{6}^{\prime}(15), A_{7}^{\prime}(16), A_{8}^{\prime}(17)\right), A_{4}^{\prime}(18)\left(A_{5}^{\prime}(19), A_{6}^{\prime}(20), A_{7}^{\prime}(21), A_{8}^{\prime}(22)\right)\right) ; \\
S_{2}=(0) A_{5}^{\prime}(23)\left(A_{10}^{\prime}(24)\left(A_{12}^{\prime}(25)\left(A_{14}^{\prime}(26), A_{15}^{\prime}(27)\right), A_{13}^{\prime}(28)\left(A_{14}^{\prime}(29), A_{15}^{\prime}(30)\right)\right),\right. \\
\left.A_{11}^{\prime}(31)\left(A_{12}^{\prime}(32)\left(A_{14}^{\prime}(33), A_{15}^{\prime}(34)\right), A_{13}^{\prime}(35)\left(A_{14}^{\prime}(36), A_{15}^{\prime}(37)\right)\right)\right), \\
A_{6}^{\prime}(38)\left(A_{10}^{\prime}(39)\left(A_{12}^{\prime}(40)\left(A_{14}^{\prime}(41), A_{15}^{\prime}(42)\right), A_{13}^{\prime}(43)\left(A_{14}^{\prime}(44), A_{15}^{\prime}(45)\right)\right),\right. \\
A_{11}^{\prime}(46)\left(A_{12}^{\prime}(47)\left(A_{14}^{\prime}(48), A_{15}^{\prime}(49)\right), A_{13}^{\prime}(50)\left(A_{14}^{\prime}(51), A_{15}^{\prime}(52)\right)\right), \\
A_{7}^{\prime}(53)\left(A_{10}^{\prime}(54)\left(A_{12}^{\prime}(55)\left(A_{14}^{\prime}(56), A_{15}^{\prime}(57)\right), A_{13}^{\prime}(58)\left(A_{14}^{\prime}(59), A_{15}^{\prime}(60)\right)\right),\right. \\
\left.A_{11}^{\prime}(61)\left(A_{12}^{\prime}(62)\left(A_{14}^{\prime}(63), A_{15}^{\prime}(64)\right), A_{13}^{\prime}(65)\left(A_{14}^{\prime}(66), A_{15}^{\prime}(67)\right)\right)\right), \\
A_{9}^{\prime}(68)\left(A_{10}^{\prime}(69)\left(A_{12}^{\prime}(70)\left(A_{14}^{\prime}(71), A_{15}^{\prime}(72)\right), A_{13}^{\prime}(73)\left(A_{14}^{\prime}(74), A_{15}^{\prime}(75)\right)\right),\right. \\
\left.A_{11}^{\prime}(76)\left(A_{12}^{\prime}(77)\left(A_{14}^{\prime}(78), A_{15}^{\prime}(79)\right), A_{13}^{\prime}(80)\left(A_{14}^{\prime}(81), A_{15}^{\prime}(82)\right)\right)\right) .
\end{gathered}
$$


3) 第 4 步相似连接之后增加了以下连接:

(19) $A_{10}^{\prime}(24) ;(19) A_{11}^{\prime}(31) ;(20) A_{10}^{\prime}(39) ;(20) A_{11}^{\prime}(46) ;(21) A_{10}^{\prime}(54) ;(21) A_{11}^{\prime}(61) ;(22) A_{10}^{\prime}(69) ;(22) A_{11}^{\prime}(76)$.

4) 集合内部的补充状态.

有些集合内部全部是一个字符, 不需要补充状态, 例如 “(0) $A_{1}^{\prime}(1) ”=“(0)\{$ 封, F, 丰, 峰 $\}(1) ”$, 相当于 “(0) 封 (1)”, “(0)F(1)”, “(0) 丰 (1)”, 及 “(0) 峰 (1)”.

有些集合内部出现不只是一个字符, 需要补充状态, 例如, “(12) $A_{3}^{\prime}(13) ”="(12)\{$ 伸, shen, 深, 神 $\}$ (13)”, 相当于 “(12) 伸 (13)”, “(12) \{深\}(13)”, “(12) 神 (13)”及 “(12) $\{\mathrm{s}(83) \mathrm{h}(84) \mathrm{e}(85) \mathrm{n}\}(13) ” ;$ 注意, “(12) $\operatorname{shen}(13)$ ” 的输入字符不止一个, 需要补充状态, 成为 “(12) $\{\mathrm{s}(83) \mathrm{h}(84) \mathrm{e}(85) \mathrm{n}\}(13)$ ”.

5) V- 不确定的处理. 例如, 3 个 V- 不确定串的交错, “封 DE 榜伸 $\mathrm{f}$ 丰 $\mathrm{g}$ 德神 Ban HI Gu 事 J”, 其中, 字符串 $S_{1}=A_{1} A_{2} A_{3}, S_{2}=B_{1} B_{2} B_{3} B_{4}, S_{3}=C_{1} C_{2} C_{3}, A_{i} \in \Omega, B_{i} \in \Omega, C_{i} \in \Omega, i=1,2,3,4, \mathrm{D}$, $\mathrm{E}, \mathrm{f}, \mathrm{g}, \mathrm{H}, \mathrm{I} \in \Omega, \Omega$ 是给定的符号集.

多完全自动机工作如下.

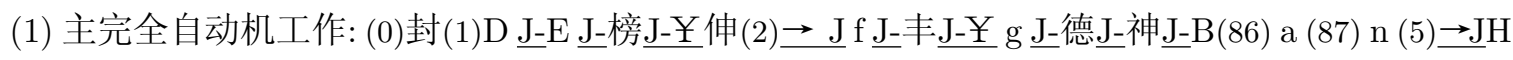

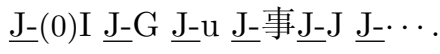

(2) 第一个¥启动第 1 个副完全自动机工作:

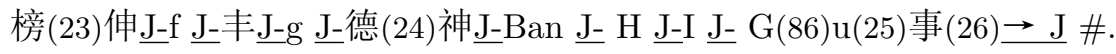

(3) 第二个 $¥$ 启动第 2 个副完全自动机工作: 丰(1)g.J-德吕神(2)B(87) a (88) n (5) $\rightarrow$ J\#, 其中, J-表 示计数器减 $1, \rightarrow \mathrm{J}$ 表示计数器置确定值, ¥启动自动机, \# 关闭该副自动机, 半匹配成功.

\section{6 结束语}

本文给出一个检测巨量 U- 不确定字符串及巨量 V- 不确定字符串和巨量 U-V- 不确定字符串的 快速匹配完全自动机的生成方法, 包括两两不相交化、无同源后续奇点化、交错情况的并行处理, 给 出并行完全自动机的台数, 并指出通常正则表达式匹配可能出现相似连接和交错情况的两种遗漏, 而 且如果没有从整体的角度对 U- 不确定串中的字符子串集进行两两不相交化及无同源后续奇点化的处 理, 结果, 就可能出现错误或者状态数目的增多.

\section{参考文献}

1 Aho A, Corasick M. Efficient string matching: an aid to bibliographic search. Commun ACM, 1975, 18: 333-340

2 Navarro G, Raffitnot M. Flexible Pattern Matching in Strings: Practical On-line Search Algorithms for Texts and Biological Sequences. Cambridge: Cambridge University Press, 2002

3 Alicherry M, Muthuprasanna M, Kumar V. High speed pattern matching for network IDS/IPS. In: Proc IEEE International Conference Network Protocols. Santa Barbara, 2006. 187-196

4 Baker Z, Prasanna V. High-throughput linked-pattern matching for intrusion detection systems. In: Proceedings of the 2005 ACM symposium on Architecture for Networking and Communications systems, 2005. 193-202

5 Baker Z K, Prasanna V K. Time and area efficient pattern matching on FPGAs. In: Proc FPGA, 2004. 223-232

6 Bispo J, Sourdis I, Cardoso J M, et al. Regular expression matching for reconfigurable packet inspection. In: Proceedings of IEEE International Conference on Field Programmable Technology, 2006. 119-126

7 Clark C R, Schimmel D E. Scalable pattern matching for high speed networks. In: Proc FCCM, 2004. 249-257 
8 Dharmapurikar S, Lockwood J W. Fast and scalable pattern matching for network intrusion detection systems. IEEE J Sel Area Ommunic, 2006, 24: 1781-1792

9 Knuth D. The Art of Computer Programming. Vol 3. Sorting and Searching. New York: Addison-Wesley, 1973

10 He L T, Fang B X, Yu X Z. A time optimal exact string matching algorithm (in Chinese). J Softw, 2005, 16: 676-683

11 Kumar S, Dharmapurikar S, Yu F. Algorithms to accelerate multiple regular expressions matching for deep packet inspection. In: SIGCOMM, 2006. 339-350

12 Liu Y B, Yang Y F, Liu P, et al. A table compression method for extended Aho-Corasick automaton. In: Proc CIAA, 2009. 84-93

13 Liu P. Research of string matching for Internet content filtering (in Chinese). Master Thesis. Beijing: Institute of Computing Technology, Chinese Academy of Sciences. 2005

14 Nieminen J, Kilpel P. Efficient implementation of Aho-Corasick pattern matching automata using unicode. Softw Pract Exper, 2007, 37: 669-690

15 Nishimuar T, Fukamachi S, Shinohara T. Speed-up of Aho-Corasick pattern matching machines by rearranging states. In: SPIRE, 2001. 175-185

16 Papaefstathiou I, Dimopoulos V, Pnevmatikatos D. A memory-efficient reconfigurable Aho-Corasick FSM implementation for intrusion detection systems. In: IEEE ICSAMOS, 2007. 186-193

17 Piyachon P, Luo Y. Efficient memory utilization on network processors for deep packet inspection. In: ANCS, 2006. $71-80$

18 Rejeb J, Srinivasan M. Extension of Aho-Corasick algorithm to detect injection attacks. In: Advances in Computer and Information Sciences and Engineering. Berlin/Heidelberg: Springer Verlag, 2008. 207-212

19 Song H, Dai Y Q. A new fast string matching algorithm for content filtering and detection (in Chinese). J Comput Res Dev, 2004, 41: 940-948

20 Sourdis I, Pnevmatikatos D N, Vassiliadis S. Scalable multigigabit pattern matching for packet inspection. IEEE Trans VLSI Syst, 2008, 16: 156-166

21 Tan L, Brotherton B, Sherwood T. Bit-split string-matching engines for intrusion detection and prevention. ACM Trans Architect Code Optimiz (TACO), 2006, 3: 3-34

22 Tripp G. A finite-state machine based string matching system for intrusion detection on high-speed networks. In: Proc EICAR, 2005. 26-40

23 Tseng K K, Lai Y C, Lin Y D, et al. A fast scalable automaton matching accelerator for embedded content processors. ACM Trans Embed Comput Syst, 2008, 8: 38-43

24 Tuck N, Sherwood T, Calder B, et al. Deterministic memory efficient string matching algorithms for intrusion detection. In: Proc IEEE Infocom, 2004. 333-340

25 Yu J M, Xue Y B, Li J. Memory efficient string matching algorithm for network intrusion management system. Tsinghua Sci Technol, 2007, 12:585-593

$26 \mathrm{Hu}$ Y, Wang P F, Hwang K. A fast algorithm for multi-string matching based on automata optimization. In: IEEE International Conference on Future Computer and Communication (ICFCC), 2010. 379-383

\title{
Giant complete automaton for uncertain multiple string matching and its high speed construction algorithm
}

\author{
HU Yue ${ }^{1,2 *}$, GAO QingShi ${ }^{1,2}$, GUO Li $^{2} \&$ WANG PeiFeng ${ }^{1}$ \\ 1 School of Information Engineering, University of Science and Technology Beijing, Beijing 100083, China; \\ 2 Institute of Computer Technology, Chinese Academy of Sciences, Beijing 100190, China \\ *E-mail: huhuyue_001@sina.com
}

Abstract Multiple string matching is often completed under the presence of U- or V-uncertain-strings, or a 
combination of the two. Recognizing large numbers of strings with U-, V-, and U-V-uncertain-strings, including the interleaving of two or more uncertain strings, is the key to the successful detection of harmful information. This paper proposes a complete automaton and its high speed construction algorithm to detect large-scale U-, $\mathrm{V}$-, and U-V-uncertain multiple strings, including two or more uncertain strings interlaced with one another. The maximum of the parallel complete automaton of the V-uncertain string is also reported. Finally, this study reveals that two kinds of pretermissions, a similarly connected pretermission and interlaced string pretermission, may appear in the matching of the regular expressions. The result of this maybe mistake or the number of states in the automaton may be increased, if the intersection of the U-uncertain strings sets, and the "homologous subsequent especial point" in the U-uncertain strings sets, never be eliminated from whole system.

Keywords multiple string matching, U-uncertain-strings, V-uncertain-strings, U-V-uncertain-strings, complete automaton 